\title{
Corrigendum
}

\section{Corrigendum to "Comparative Aspects of Spin-Dependent Interaction Potentials for Spin-1/2 and Spin-1 Matter Fields"}

\author{
P. C. Malta, ${ }^{1,2}$ L. P. R. Ospedal, ${ }^{2}$ K. Veiga, ${ }^{2,3}$ and J. A. Helayël-Neto ${ }^{2}$ \\ ${ }^{1}$ Institut für Theoretische Physik, University of Heidelberg, Philosophenweg 16, 69120 Heidelberg, Germany \\ ${ }^{2}$ Centro Brasileiro de Pesquisas Físicas (CBPF), Rua Dr. Xavier Sigaud 150, Urca, 22290-180 Rio de Janeiro, RJ, Brazil \\ ${ }^{3}$ Instituto Federal de Educação, Ciência e Tecnologia da Bahia, Campus Vitória da Conquista, Avenida Amazonas 3150, \\ Zabelê, 45075-265 Vitória da Conquista, BA, Brazil
}

Correspondence should be addressed to P. C. Malta; malta@thphys.uni-heidelberg.de

Received 20 October 2016; Accepted 12 February 2017; Published 19 April 2017

Copyright ( 2017 P. C. Malta et al. This is an open access article distributed under the Creative Commons Attribution License, which permits unrestricted use, distribution, and reproduction in any medium, provided the original work is properly cited. The publication of this article was funded by SCOAP ${ }^{3}$.

\section{Introduction}

In the article titled "Comparative Aspects of Spin-Dependent Interaction Potentials for Spin-1/2 and Spin-1 Matter Fields" [1], calculations for some interparticle potentials for sources with spin-1/2 and spin-1 in the nonrelativistic (NR) regime contained inadvertent errors which should be corrected as follows. The main conclusions are unaltered.

We note further that we have included contact terms coming from Dirac delta contributions of the following Fourier transforms:

$$
\begin{aligned}
& \int \frac{d^{3} \vec{q}}{(2 \pi)^{3}} \frac{\vec{q}_{i} \vec{q}_{j}}{\vec{q}^{2}+m^{2}} e^{i \vec{q} \cdot \vec{r}} \\
& =\frac{\delta_{i j}}{3} \delta^{(3)}(\vec{r}) \\
& \quad+\frac{e^{-m r}}{4 \pi r^{3}}\left[\delta_{i j}(1+m r)-\widehat{r}_{i} \widehat{r}_{j}\left(m^{2} r^{2}+3 m r+3\right)\right], \\
& \int \frac{d^{3} \vec{q}}{(2 \pi)^{3}} \frac{\vec{q}^{2}}{\vec{q}^{2}+m^{2}} e^{i \vec{q} \cdot \vec{r}}=\delta^{(3)}(\vec{r})-\frac{m^{2} e^{-m r}}{4 \pi r},
\end{aligned}
$$

which are the massive generalizations of the "massless" results from [2].

In what follows we list the corrections, indicated by $\Delta V$, that must be added to some of the potentials given in [1]. The mass of the mediator, $m$, corresponds to either $m_{\phi}$ or $m_{A}$, as the case may be. We also highlight that not all the potentials from [1] are affected and in this note we only indicate those which are modified (Moreover, we need to use $\left(S_{i}\right)_{j k}=-i \epsilon_{i j k}$ in all potentials. In [1], we had the opposite sign, so one must make $\vec{S} \rightarrow-\vec{S}$ throughout.).

\section{Correcting the Potentials}

2.1. Potentials (Spin-1/2). The $S-P S$ and $V-P V$ potentials from [1] are not affected. The other potentials receive partial corrections. For convenience, we define the function:

$$
I(r) \equiv \frac{1}{4}\left(\frac{1}{m_{1}^{2}}+\frac{1}{m_{2}^{2}}\right)\left(\vec{p}^{2}-\frac{m^{2}}{4}\right) \frac{e^{-m r}}{4 \pi r}
$$

so that the potentials in equations (25), (30) (As mentioned in equation (30) in [1], the PS-PS potential for $s=1 / 2$ has the same functional form as the PS-PS potential for $s=1$.), (44), and (45) in [1] receive the respective corrections:

$$
\begin{aligned}
& \Delta V_{S-S}^{s=1 / 2}=g_{S}^{1} g_{S}^{2} \delta_{1} \delta_{2} I(r), \\
& \Delta V_{P S-P S}^{s=1 / 2}=-g_{P S}^{1} g_{P S}^{2} \frac{\langle\vec{\sigma}\rangle_{1} \cdot\langle\vec{\sigma}\rangle_{2}}{12 m_{1} m_{2}} \delta^{(3)}(\vec{r}), \\
& \Delta V_{V-V}^{s=1 / 2}=-e_{1} e_{2}\left\{\delta_{1} \delta_{2} I(r)\right. \\
& \left.\quad+\left[\frac{\delta_{1} \delta_{2}}{8}\left(\frac{1}{m_{1}^{2}}+\frac{1}{m_{2}^{2}}\right)+\frac{\langle\vec{\sigma}\rangle_{1} \cdot\langle\vec{\sigma}\rangle_{2}}{6 m_{1} m_{2}}\right] \delta^{(3)}(\vec{r})\right\},
\end{aligned}
$$




$$
\begin{gathered}
\Delta V_{P V-P V}^{s=1 / 2}=+g_{P V}^{1} g_{P V}^{2}\langle\vec{\sigma}\rangle_{1} \cdot\langle\vec{\sigma}\rangle_{2}\{I(r) \\
\left.+\left[\frac{1}{8}\left(\frac{1}{m_{1}^{2}}+\frac{1}{m_{2}^{2}}\right)-\frac{1}{m^{2}}\right] \frac{\delta^{(3)}(\vec{r})}{3}\right\} .
\end{gathered}
$$

Equation (4) modifies the full potential by eliminating a momentum-independent monopole-monopole term $m^{2}\left(1 / m_{1}^{2}+1 / m_{2}^{2}\right)\left(e^{-m r} / 4 \pi r\right)$. A similar modification, but momentum-dependent, is induced by (6). For the other potentials, the modifications involve spin-spin interactions, mostly coupled to contact terms.

\subsection{Potentials (Spin-1)}

2.2.1. Vector Representation. The corrections to equations (28), (30), (47), and (48) in [1] are, respectively:

$$
\begin{gathered}
\Delta V_{S-S}^{s=1}=\frac{g_{S}^{1} g_{S}^{2}}{2} \frac{\delta_{1} \delta_{2}}{m_{1} m_{2}}\left\{I(r)-\frac{1}{48}\left(\frac{1}{m_{1}^{2}}+\frac{1}{m_{2}^{2}}\right)\right. \\
\left.\cdot \delta^{(3)}(\vec{r})\right\}, \\
\Delta V_{P S-P S}^{s=1}=-g_{P S}^{1} g_{P S}^{2} \frac{\langle\vec{S}\rangle_{1} \cdot\langle\vec{S}\rangle_{2}}{3} \delta^{(3)}(\vec{r}), \\
\Delta V_{V-V}^{s=1}=-e_{1} e_{2}\left\{2 \delta_{1} \delta_{2} I(r)\right. \\
+\left[\frac{2}{3} \frac{\langle\vec{S}\rangle_{1} \cdot\langle\vec{S}\rangle_{2}}{m_{1} m_{2}}+\frac{\delta_{1} \delta_{2}}{6}\left(\frac{1}{m_{1}^{2}}+\frac{1}{m_{2}^{2}}\right)\right] \\
\left.\cdot \delta^{(3)}(\vec{r})\right\}, \\
\Delta V_{P V-P V}^{s=1}=g_{P V}^{1} g_{P V}^{2} \frac{\langle\vec{S}\rangle_{1} \cdot\langle\vec{S}\rangle_{2}}{12 m_{1} m_{2}} \delta^{(3)}(\vec{r})
\end{gathered}
$$

whereby, in (10), $I(r)$ cancels another term in (47) from [1].

2.2.2. Tensor Representation. The correction to the PS-PS potential in this representation is $\Delta V_{P S-P S}^{s=1} / 4 m_{1}^{2} m_{2}^{2}$ (cf. (9)). Also, the correction to the $P V-P V$ potential in this representation is the same as in (11).

In [1], equations (65) receive the respective corrections:

$$
\begin{aligned}
& \Delta V_{S-S}^{s=1}=\frac{g_{S}^{1} g_{S}^{2}}{2} \frac{\delta_{1} \delta_{2}}{m_{1} m_{2}}\left\{I(r)-\frac{5}{48}\left(\frac{1}{m_{1}^{2}}+\frac{1}{m_{2}^{2}}\right)\right. \\
& \left.\cdot \delta^{(3)}(\vec{r})\right\},
\end{aligned}
$$

$$
\begin{aligned}
& \Delta V_{V-V}^{s=1}=-e_{1} e_{2}\left\{2 \delta_{1} \delta_{2} I(r)\right. \\
& \left.+\left[\frac{2}{3} \frac{\langle\vec{S}\rangle_{1} \cdot\langle\vec{S}\rangle_{2}}{m_{1} m_{2}}+\frac{\delta_{1} \delta_{2}}{3}\left(\frac{1}{m_{1}^{2}}+\frac{1}{m_{2}^{2}}\right)\right] \delta^{(3)}(\vec{r})\right\} .
\end{aligned}
$$

We note that the correction in (13) excludes one momentum-dependent term associated with $\vec{p}^{2}\left(1 / m_{1}^{2}+\right.$ $\left.1 / m_{2}^{2}\right)\left(e^{-m r} / 4 \pi r\right)$ in the $V-V$ potential in equation (65) from [1].

\section{Concluding Remarks}

We have presented the corrections to some of the results given in [1]. The corrections introduced affect mostly the monopole-monopole sectors (e.g., (4), (8), and (12)).

The conclusions presented in [1] remain unaltered: in general, the interparticle potentials associated with NR spin$1 / 2$ and spin-1 sources present similarities, especially, in the spin-spin sectors of the $S-S, S-P S, P S-P S$, and $V-V$ interactions. The most important modifications are due to the presence of contact terms, which do not play a role in macroscopic interactions.

\section{References}

[1] P. C. Malta, L. P. R. Ospedal, K. Veiga, and J. A. Helayël-Neto, "Comparative aspects of spin-dependent interaction potentials for spin-1/2 and spin-1 matter fields," Advances in High Energy Physics, vol. 2016, Article ID 2531436, 13 pages, 2016.

[2] G. S. Adkins, "Three-dimensional Fourier transforms, integrals of spherical Bessel functions, and novel delta function identities," https://arxiv.org/abs/1302.1830. 

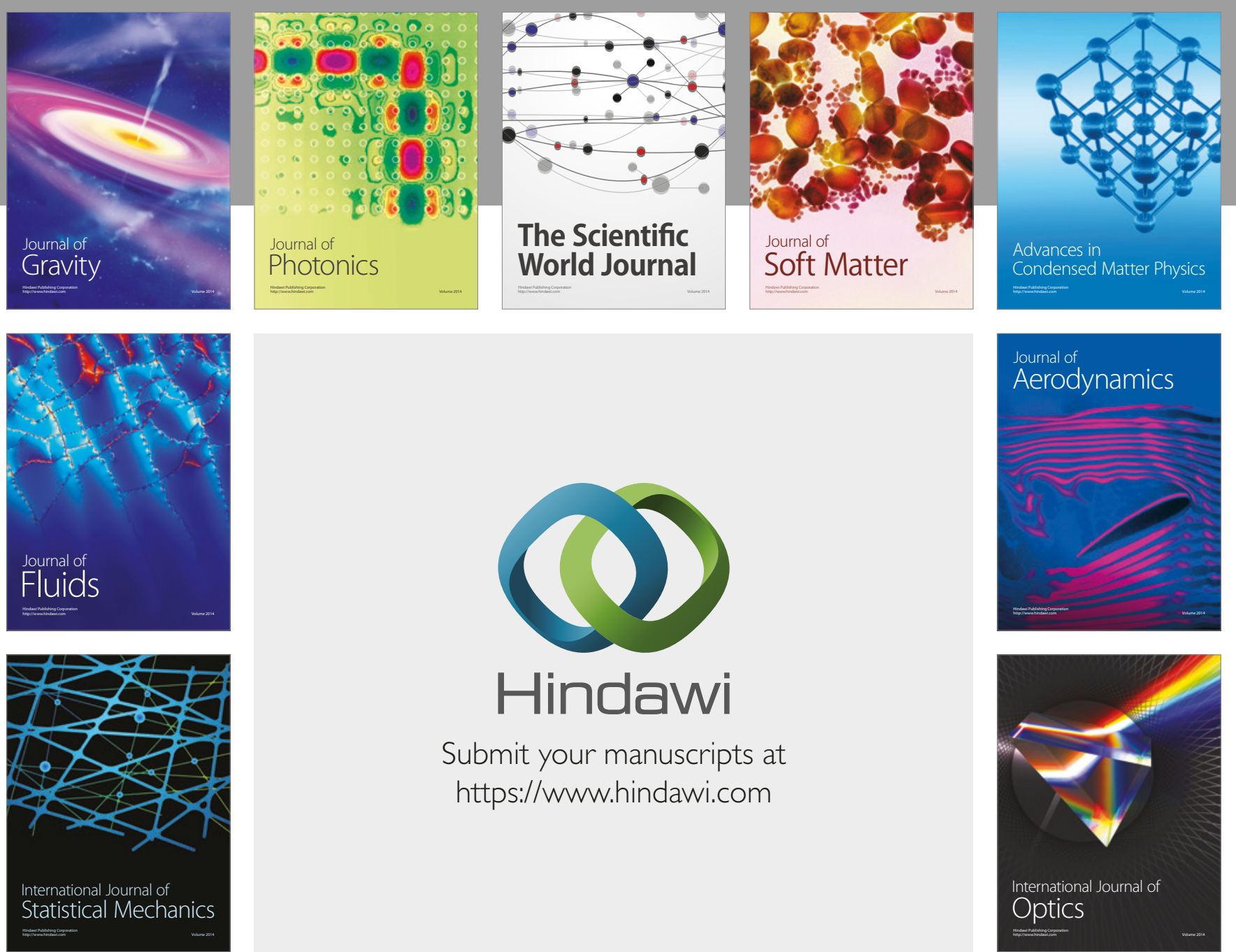

Submit your manuscripts at

https://www.hindawi.com
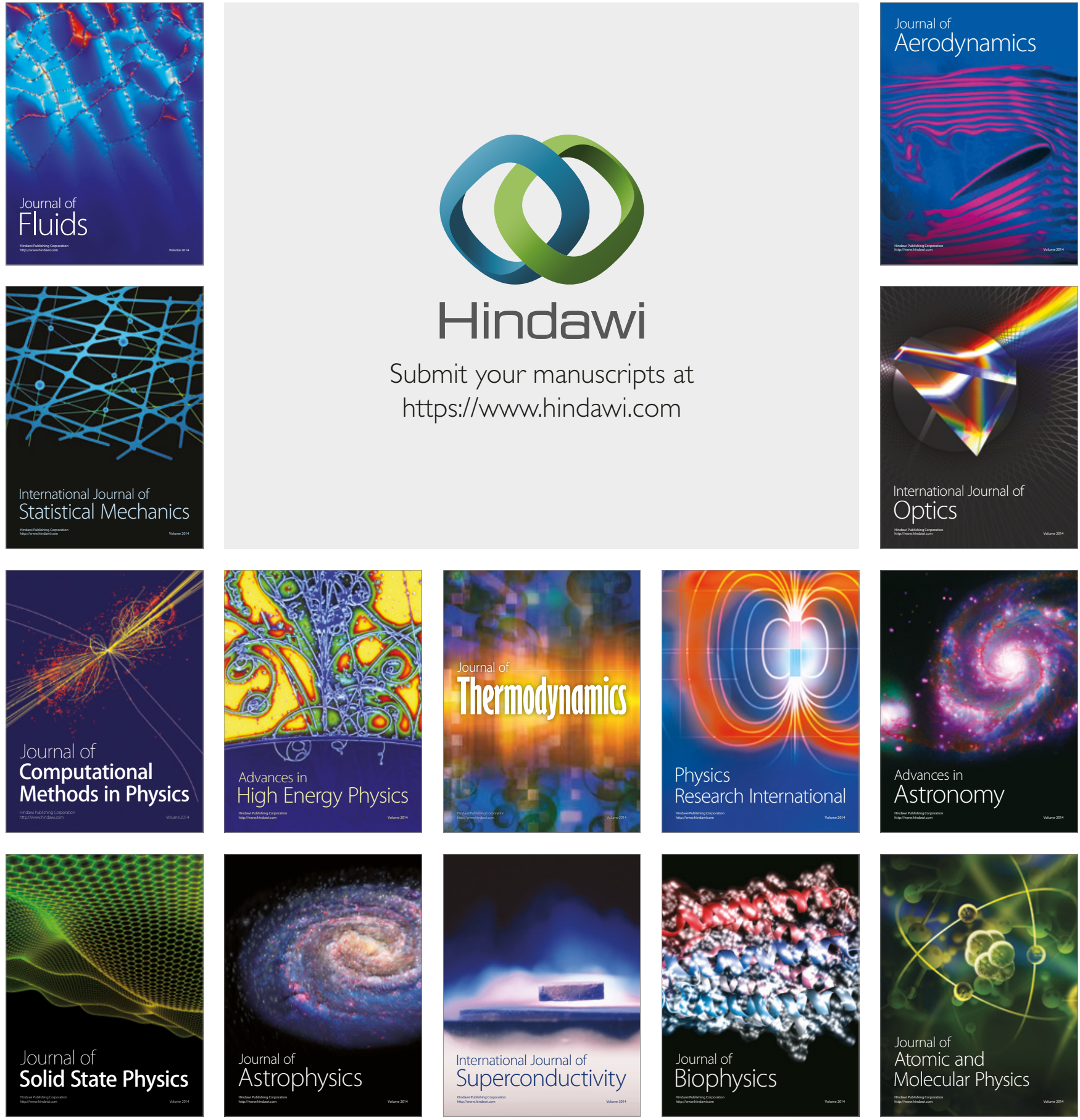